\title{
Un dernier Et encore
}

\section{Bruno Kesseli}

Dr méd. et lic. phil., (ancien) rédacteur en chef

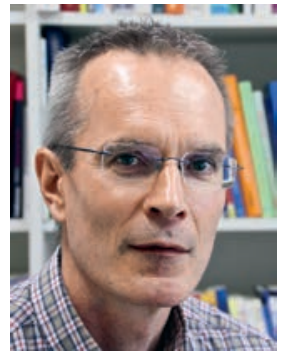

«Mais qu'est-ce qu'il fait encore ici celui-là?», vous demanderez-vous peut-être en tombant sur cette page. La revue ne lui a-t-elle pas fait ses adieux en grande pompe il y a un mois?

C'est tout à fait exact. Certes, je reste officiellement le rédacteur en chef du BMS jusqu'à la fin juillet, mais si vous envoyez un mail à mon ancienne adresse bkesseli@emh.ch, vous recevrez un message d'adieu. Dans les faits, Matthias Scholer a repris mes fonctions début juin, en raison des jours de congé que j'ai cumulés au fil des ans. Vous connaissez certainement cela. Lédition thématique consacrée à mon départ - un coup de maître organisé par la rédaction de Muttenz et de nombreux compagnons de longue date - m'a surpris autant que vous, pour ne pas dire que j'en suis tombé de ma chaise. Elle montre non seulement que les journalistes professionnels sont tout aussi capables de garder le secret que les médecins, mais elle témoigne en outre d'une estime qui m'impressionne beaucoup et me fait infiniment plaisir. Et le numéro 2324/2019 me rappellera toujours que ma décision de rejoindre le BMS en 2005 était la bonne.

Toutefois, cette édition d'adieu a aussi compliqué un peu mon dernier article en tant que rédacteur en chef du BMS. Cet «Et encore» était en effet programmé depuis longtemps et presque complètement rédigé dans ma tête. Mes collègues m'ont pris de vitesse, résumant parfaitement mes propos de départ par anticipation, que ce soit dans l'éditorial de Jürg Schlup, l'hommage d'Eberhard Wolff, le «Et encore» de la direction des EMH ou les nombreux articles courts. Celles et ceux qui ne connaissaient pas déjà le "modèle dual», qui fait à mes yeux la force et la spécificité du BMS, sont certainement renseignés depuis.

Je dois donc improviser. Cela m'est d'autant plus facile que mes petits cachotiers se sont occupés pour moi de la partie complexe - dresser le bilan de près de 14 années au service du BMS. Ma personne et mon travail ont été présentés sous un jour très favorable, mais je ne m'en plains pas. En tant que lectrices et lecteurs, vous saurez relativiser les louanges et un peu de narcissisme est sans doute admissible lorsque l'on s'en va.

Qu'est-ce qu'il me reste en dehors de nombreux beaux souvenirs et d'une certaine nostalgie? La réponse est simple: une immense gratitude. J'aimerais profiter de l'occasion pour l'exprimer ici. Depuis que j'ai pris mes fonctions de rédacteur en chef en novembre 2005, j’ai toujours pu compter sur une équipe formidable à Muttenz, qui m'a soutenu avec compétence et sans qui je n'aurais pas pu réussir mon grand écart entre le travail rédactionnel et mes activités au cabinet.

A ce titre, je dois absolument citer (dans leur ordre d'arrivée) Margrit Neff, Thomas Heuer, Annette Eichholtz, Elisa Jaun, Isabel Zwyssig et Tanja Kühnle. Un grand merci également aux actuels membres de la rédaction externe Erhard Taverna, Hans Stalder, Jean Martin, Werner Bauer, Samia Hurst, Rouven Porz, Christina Aus der Au, Eberhard Wolff, Iris Ritzmann, Milo Puhan

Qu'est-ce qu'il me reste en dehors de nombreux beaux souvenirs et d'une certaine nostalgie? La réponse est simple: une immense gratitude.

et Ursina Pally pour l'excellente collaboration, ainsi qu'aux anciens membres Anna Sax, Hanspeter Kuhn, Christoph Rehmann-Sutter et Lazare Benaroyo. Mes collègues de la direction, Natalie Marty et Karin Würz, ainsi que mes chefs Ruedi Bienz et plus récemment Sandra Ziegler, m'ont témoigné une confiance inconditionnelle, tout comme la FMH et le conseil d'administration d'EMH avec le président Hans Kurt et son prédécesseur Ludwig T. Heuss. Je me dois également de mettre en lumière la collaboration avec l'actuel président de la FMH Jürg Schlup et la responsable de la communication Charlotte Schweizer. A mes yeux, elle n'aurait pas pu mieux se passer. Mon successeur Matthias Scholer, pour l'élection duquel je félicite les organes compétents des EMH et de la FMH, ainsi que vous, chers lectrices et lecteurs, est depuis des années un ami avec lequel je peux échanger d'égal à égal.

Enfin, je tiens également à vous remercier vous, chers lectrices et lecteurs. Beaucoup d'entre vous sont aussi nos auteures et auteurs, et à ce titre, coresponsables des contenus et de la qualité du BMS. Les échanges quotidiens intensifs avec vous ont constitué un élément central de mon travail et ont (presque toujours) été une source de plaisir et de satisfaction. Je me réjouis à présent de consacrer à nouveau toute mon attention professionnelle à mes patientes et patients. Pour autant, je ne cesserai pas complètement d'écrire ni ne quitterai totalement le BMS et la FMH, dont je resterai simple lecteur et simple membre.

A la revoyure! 